\title{
TAUBERIAN THEOREMS AND STABILITY OF SOLUTIONS OF THE CAUCHY PROBLEM
}

\author{
CHARLES J. K. BATTY, JAN VAN NEERVEN, AND FRANK RÄBIGER
}

Abstract. Let $f: \mathbb{R}_{+} \rightarrow X$ be a bounded, strongly measurable function with values in a Banach space $X$, and let $i E$ be the singular set of the Laplace transform $\tilde{f}$ in $i \mathbb{R}$. Suppose that $E$ is countable and $\alpha\left\|\int_{0}^{\infty} e^{-(\alpha+i \eta) u} f(s+u) d u\right\|$ $\rightarrow 0$ uniformly for $s \geq 0$, as $\alpha \searrow 0$, for each $\eta$ in $E$. It is shown that

$$
\left\|\int_{0}^{t} e^{-i \mu u} f(u) d u-\widetilde{f}(i \mu)\right\| \rightarrow 0
$$

as $t \rightarrow \infty$, for each $\mu$ in $\mathbb{R} \backslash E$; in particular, $\|f(t)\| \rightarrow 0$ if $f$ is uniformly continuous. This result is similar to a Tauberian theorem of Arendt and Batty. It is obtained by applying a result of the authors concerning local stability of bounded semigroups to the translation semigroup on $B U C\left(\mathbb{R}_{+}, X\right)$, and it implies several results concerning stability of solutions of Cauchy problems.

\section{INTRODUCTION}

Let $\mathbf{T}=\{T(t): t \geq 0\}$ be a $C_{0}$-semigroup, with generator $A$, on a Banach space $X$. For $x$ in $X$, the orbit $f(t):=T(t) x$ has Laplace transform given by the resolvent $\widetilde{f}(\lambda)=R(\lambda, A) x$ for $\operatorname{Re} \lambda>\omega$, where $\omega$ is the growth bound of the semigroup. Thus Laplace transform theory often has implications for semigroup theory. On the other hand, one can sometimes apply semigroup results to translations on suitable function spaces to recover information about Laplace transforms.

In this situation, Tauberian theorems relating Abel, Cesàro and standard convergence of $f$ produce results about the long-time asymptotic behaviour of orbits of semigroups. A simple example of this is a special case of a Tauberian theorem of Ingham [17], for which Korevaar [20] has given an elegant proof by contour integration, and which immediately gives the result that if $\mathbf{T}$ is a bounded semigroup and $\sigma(A) \cap i \mathbb{R}$ is empty, then $\left\|T(t) A^{-1}\right\| \rightarrow 0$ as $t \rightarrow \infty$. A more complicated version of this, initiated in [1], provides an estimate which was exploited in [2] both to establish a more general Tauberian theorem and to show that if $\mathbf{T}$ is a bounded semigroup, $\sigma(A) \cap i \mathbb{R}$ is countable and $\sigma_{p}\left(A^{*}\right) \cap i \mathbb{R}$ is empty, then $\|T(t) x\| \rightarrow 0$ as

Received by the editors February 12, 1996 and, in revised form, September 6, 1996.

1991 Mathematics Subject Classification. Primary 44A10; Secondary 47D06, 47 D03.

Key words and phrases. Laplace transform, Tauberian theorem, singular set, countable, $C_{0^{-}}$ semigroup, stability, local spectrum, orbit, Cauchy problem.

The work on this paper was done during a two-year stay at the University of Tübingen. Support by an Individual Fellowship from the Human Capital and Mobility Programme of the European Community is gratefully acknowledged. I warmly thank Professor Rainer Nagel and the members of his group for their hospitality (second author). It is part of a research project supported by the Deutsche Forschungsgemeinschaft DFG (third author). Work in Oxford was also supported by an EPSRC Visiting Fellowship Research Grant (first and third authors). 
$t \rightarrow \infty$, for each $x$ in $X$. In this case, the Tauberian theorem and the semigroup theorem, although derived from the same source, are independent results (see the survey articles [6] and [7] for accounts of developments of the two themes). The semigroup theorem was obtained independently by Lyubich and Vũ Quôc Phóng [23], using a completely different functional-analytic method.

For semigroups which are not uniformly bounded, a few results have been obtained [2], [4], [5], [10], [12], showing that $\|T(t) x\| \rightarrow 0$ for an individual vector $x$, assuming that the orbit is bounded and/or uniformly continuous, and also assuming global spectral conditions. In a different development using both complexanalytic and functional-analytic techniques, some complementary results have been obtained for bounded semigroups, where the spectral conditions apply on a densely embedded subspace [16], [8]. Recently, we obtained a result for individual orbits of bounded semigroups, where the spectral condition is truly local as it applies only to that orbit [9].

In this paper, we reverse the usual direction of flow between Tauberian theorems and asymptotic behaviour of semigroups, and we also unify the two strands described in the last paragraph. We establish that the results of [9] can be applied to translation semigroups, by showing that the singular set of $\widetilde{f}$ in $i \mathbb{R}$ coincides with the local unitary spectrum at $f$ of the generator of the translation semigroup. We thereby obtain a new Tauberian theorem for bounded functions whose Laplace transforms have only countably many singularities on the imaginary axis (Theorem 4.3). The theorem is similar in format to [2, Theorem 4.1], but the conditions are somewhat different. The result is directly applicable to semigroups and it easily implies all the semigroup results mentioned above. In addition, it gives new results for bounded, uniformly continuous orbits of unbounded semigroups (or of ill-posed Cauchy problems), assuming only local spectral conditions (Section 5). Related results have been obtained in [4], [12], [25], [29].

In [9], we also obtained local versions of several other related theorems concerning asymptotic behaviour of bounded semigroups. Our techniques enable us to give corresponding theorems for Laplace transforms (Theorems 4.1, 4.6 and 4.7). The Tauberian theorem for Laplace transforms also implies corresponding theorems for power series, and hence local stability results for single operators (Section 6).

\section{A UNIFORM BOUND}

Throughout the paper, $X$ will be a complex Banach space, $\mathbb{R}_{+}$will be the halfline $[0, \infty), \mathbb{C}_{+}$will be the half-plane $\{z \in \mathbb{C}: \operatorname{Re} z>0\}$. For $z$ in $\mathbb{C}$ and $r>0$, we will put $B(z, r)=\{w \in \mathbb{C}:|w-z|<r\}$.

Let $f: \mathbb{R}_{+} \rightarrow X$ be strongly measurable, and let $\tilde{f}$ be the Laplace transform of $f:$

$$
\widetilde{f}(z)=\int_{0}^{\infty} e^{-t z} f(t) d t
$$

We assume that $\widetilde{f}(z)$ exists for all $z$ in $\mathbb{C}_{+}$, so $\widetilde{f}$ is holomorphic in $\mathbb{C}_{+}$(usually, $f$ will be bounded). A point $\lambda=i \eta$ in $i \mathbb{R}$ is said to be a regular point for $\tilde{f}$ if there is an open neighbourhood $U$ of $\lambda$ in $\mathbb{C}$ and a holomorphic function $g: U \rightarrow X$ such that $g(z)=\widetilde{f}(z)$ whenever $z \in U \cap \mathbb{C}_{+}$. The singular set $i E$ of $\widetilde{f}$ is the set of all points of $i \mathbb{R}$ which are not regular points. 
For $s \geq 0$, we shall use the notation $f_{s}$ to denote the translate of $f$, so $f_{s}(t)=$ $f(s+t)\left(t \in \mathbb{R}_{+}\right)$.

Let $f: \mathbb{R}_{+} \rightarrow X$ be bounded and strongly measurable. The old result of Ingham [17, Theorem I], or the special case proved in a simple way by Korevaar [20, p.113], shows that, for each regular point $i \eta$ of $\widetilde{f}$ in $i \mathbb{R}$,

$$
\sup _{s \geq 0}\left\|\int_{0}^{s} e^{-i \eta t} f(t) d t\right\|<\infty
$$

As we shall see in the first paragraph of the proof of Proposition 2.1, this is essentially the same as saying that $\sup _{s \geq 0}\left\|g_{s}(i \eta)\right\|<\infty$, where $g_{s}$ is a holomorphic extension of $\tilde{f}_{s}$. We shall need to extend such uniform bounds into the left halfplane. We do this in the following proposition, using an old technique involving factors such as $1+z^{2} / r^{2}$, which has already been employed many times in this area $[20],[1],[2],[16],[8],[9]$.

Proposition 2.1. Let $f: \mathbb{R}_{+} \rightarrow X$ be bounded and strongly measurable, let $E$ be the singular set of $\widetilde{f}$ and let $g: V \rightarrow X$ be a holomorphic extension of $\widetilde{f}$ to a connected open neighbourhood $V$ of $\mathbb{C}_{+} \cup i(\mathbb{R} \backslash E)$. For each $s \geq 0, \widetilde{f}_{s}$ has a holomorphic extension $g_{s}: V \rightarrow X$. Moreover, for each $\lambda$ in $V$, there exist a neighbourhood $U$ of $\lambda$ in $V$ and a constant $c$ such that $\left\|g_{s}(z)\right\| \leq c$ for all $s \geq 0$ and all $z$ in $U$.

Proof. For Re $z>0$,

$$
\begin{aligned}
\tilde{f}_{s}(z) & =\int_{0}^{\infty} e^{-t z} f(s+t) d t \\
& =\int_{s}^{\infty} e^{-(t-s) z} f(t) d t \\
& =e^{s z}\left(\widetilde{f}(z)-\int_{0}^{s} e^{-t z} f(t) d t\right) .
\end{aligned}
$$

Thus we may take

$$
g_{s}(z)=e^{s z}\left(g(z)-\int_{0}^{s} e^{-t z} f(t) d t\right) \quad(z \in V)
$$

as the holomorphic extension of $\widetilde{f}_{s}$.

If $\operatorname{Re} \lambda>0$, then we may take $r>0$ such that $\operatorname{Re} \lambda-2 r \geq 0$. For $z$ in $B(\lambda, r)$,

$$
\left\|g_{s}(z)\right\|=\left\|\tilde{f}_{s}(z)\right\| \leq \int_{0}^{\infty} e^{-r t}\|f(s+t)\| d t \leq \frac{\|f\|_{\infty}}{r},
$$

so we may take $U=B(\lambda, r)$ and $c=\|f\|_{\infty} / r$.

If $\lambda \in V$ and $\operatorname{Re} \lambda<0$, then we may take $r>0$ such that $2 r+\operatorname{Re} \lambda \leq 0$ and $B(\lambda, r) \subseteq V$. For $z$ in $B(\lambda, r)$,

$$
\left\|g_{s}(z)\right\| \leq M+\int_{0}^{s} e^{-r(s-t)}\|f\|_{\infty} d t \leq M+\frac{\|f\|_{\infty}}{r},
$$

where $M=\sup _{|z-\lambda| \leq r}\|g(z)\|$. Thus we may take $U=B(\lambda, r)$ and $c=M+\|f\|_{\infty} / r$.

Now suppose that $\lambda \in i \mathbb{R} \backslash i E$. Choose $r>0$ such that $\overline{B(\lambda, 2 r)} \subseteq V$. Let

$$
h_{s}(z)=\left(1+\frac{(z-\lambda)^{2}}{4 r^{2}}\right) g_{s}(z) \quad(z \in V) .
$$


For $z=\lambda+2 r e^{i \theta}$, where $-\pi / 2<\theta<\pi / 2$,

$$
\begin{aligned}
\left\|h_{s}(z)\right\| & =\left|1+e^{2 i \theta}\right|\left\|\tilde{f}_{s}(z)\right\| \\
& \leq 2 \cos \theta \int_{0}^{\infty} e^{-2 r t \cos \theta}\|f(s+t)\| d t \\
& \leq 2 \cos \theta \frac{\|f\|_{\infty}}{2 r \cos \theta} \\
& =\frac{\|f\|_{\infty}}{r} .
\end{aligned}
$$

For $z=\lambda+2 r e^{i \theta}$, where $\pi / 2<\theta<3 \pi / 2$,

$$
\begin{aligned}
\left\|h_{s}(z)\right\| & =\left|1+e^{2 i \theta}\right|\left\|e^{s z} g(z)-\int_{0}^{s} e^{(s-t) z} f(t) d t\right\| \\
& \leq 2|\cos \theta|\left(M+\int_{0}^{s} e^{2 r(s-t) \cos \theta}\|f(t)\| d t\right) \\
& \leq 2|\cos \theta|\left(M+\frac{\left(1-e^{2 r s \cos \theta}\right)\|f\|_{\infty}}{2 r|\cos \theta|}\right) \\
& \leq 2 M+\frac{\|f\|_{\infty}}{r},
\end{aligned}
$$

where $M=\sup _{|z-\lambda| \leq 2 r}\|g(z)\|$. Thus $\left\|h_{s}(z)\right\| \leq 2 M+\|f\|_{\infty} / r$ whenever $|z-\lambda|=2 r$. By the Maximum Modulus Principle, $\left\|h_{s}(z)\right\| \leq 2 M+\|f\|_{\infty} / r$ whenever $|z-\lambda| \leq 2 r$. In particular if $|z-\lambda|<r$,

$$
\left\|g_{s}(z)\right\| \leq \frac{\left\|h_{s}(z)\right\|}{\left|1+(z-\lambda)^{2} / 4 r^{2}\right|} \leq \frac{4}{3}\left(2 M+\frac{\|f\|_{\infty}}{r}\right) .
$$

We may therefore take $U=B(\lambda, r)$ and $c=\frac{4}{3}\left(2 M+\frac{\|f\|_{\infty}}{r}\right)$.

\section{The Singular SET AND THE LOCAL SPECTRUM}

In this section, we shall establish that the singular set of $\tilde{f}$ in $i \mathbb{R}$ coincides with the local unitary spectrum at $f$ of the generator of the translation semigroup on the space of bounded, uniformly continuous functions.

Let $\mathbf{T}=\{T(t): t \geq 0\}$ be a $C_{0}$-semigroup on a Banach space $X$, with generator $A$ and growth bound $\omega$, and let $x \in X$. The local unitary spectrum, $\sigma_{u}(A, x)$, of $A$ at $x$ is defined to be the set of all points $\lambda$ in $i \mathbb{R}$ such that there do not exist an open neighbourhood $U$ of $\lambda$ and a holomorphic function $g: U \cup \mathbb{C}_{+} \rightarrow X$ with $g(z)=R(z, A) x=(z I-A)^{-1} x$ for $\operatorname{Re} z>\omega$. For a bounded semigroup, $\sigma_{u}(A, x)$ is the singular set of $\widetilde{f}$, where $f(t)=T(t) x$ is a mild solution of the associated (well-posed) Cauchy problem. In Section 5, we shall return to this point in the more general context of ill-posed Cauchy problems.

Let $\mathbf{S}=\{S(t): t \geq 0\}$ be the $C_{0}$-semigroup of contractions on $B U C\left(\mathbb{R}_{+}, X\right)$ defined by

$$
S(t) f=f_{t}, \quad \text { where } f_{t}(s)=f(s+t) \quad\left(s, t \geq 0 ; f \in B U C\left(\mathbb{R}_{+}, X\right)\right) .
$$

Given $f$ in $B U C\left(\mathbb{R}_{+}, X\right)$, we shall show in Proposition 3.3 that the singular set of $\widetilde{f}$ in $i \mathbb{R}$ coincides with $\sigma_{u}(D, f)$, where $D$ is the generator of $\mathbf{S}$. 
Proposition 3.1. Let $f: \mathbb{R}_{+} \rightarrow X$ be bounded and uniformly continuous, let $i E$ be the singular set of $\tilde{f}$, and let $g: V \rightarrow X$ be a holomorphic extension of $\tilde{f}$ to a connected open set $V$ containing $\mathbb{C}_{+} \cup i(\mathbb{R} \backslash E)$. For $s \geq 0$, let $g_{s}$ be as in Proposition 2.1. Then for each $z$ in $V$, the map $s \mapsto g_{s}(z)$ is uniformly continuous.

Proof. First, suppose that $\operatorname{Re} z>0$. For $\delta>0$ and $\left|s-s^{\prime}\right|<\delta$,

$$
\begin{aligned}
\left\|g_{s}(z)-g_{s^{\prime}}(z)\right\| & =\left\|\int_{0}^{\infty} e^{-t z}\left(f(s+t)-f\left(s^{\prime}+t\right)\right) d t\right\| \\
& \leq \frac{1}{\operatorname{Re} z} \sup _{\left|t-t^{\prime}\right|<\delta}\left\|f(t)-f\left(t^{\prime}\right)\right\| \\
& \rightarrow 0 \quad \text { as } \delta \rightarrow 0 .
\end{aligned}
$$

Next, suppose that $z \in i \mathbb{R} \backslash i E$. Then

$$
g_{s}(z)=e^{s z}\left(g(z)-\int_{0}^{s} e^{-t z} f(t) d t\right) .
$$

Now, $s \mapsto e^{s z}$ is bounded and uniformly continuous. Since $t \mapsto e^{-t z} f(t)$ is bounded, $s \mapsto g(z)-\int_{0}^{s} e^{-t z} f(t) d t=e^{-s z} g_{s}(z)$ is uniformly continuous. Moreover, Proposition 2.1 shows that this map is bounded. Hence $s \mapsto g_{s}(z)$ is uniformly continuous.

Finally, suppose that $\operatorname{Re} z<0$ and $z \in V$. Then $s \mapsto e^{s z} g(z)$ is uniformly continuous, so it suffices to check that $s \mapsto \int_{0}^{s} e^{(s-t) z} f(t) d t$ is uniformly continuous. Now, for $s \geq s^{\prime} \geq 0$,

$$
\begin{aligned}
&\left\|\int_{0}^{s} e^{(s-t) z} f(t) d t-\int_{0}^{s^{\prime}} e^{\left(s^{\prime}-t\right) z} f(t) d t\right\| \\
&=\left\|\int_{0}^{s^{\prime}}\left(e^{(s-t) z}-e^{\left(s^{\prime}-t\right) z}\right) f(t) d t+\int_{s^{\prime}}^{s} e^{(s-t) z} f(t) d t\right\| \\
& \leq\left|e^{s z}-e^{s^{\prime} z}\right| \int_{0}^{s^{\prime}} e^{|\operatorname{Re} z| t}\|f(t)\| d t+\int_{s^{\prime}}^{s}\|f(t)\| d t \\
& \leq e^{s^{\prime} \operatorname{Re} z}\left|e^{\left(s-s^{\prime}\right) z}-1\right| \frac{e^{s^{\prime}|\operatorname{Re} z|}}{|\operatorname{Re} z|}\|f\|_{\infty}+\left(s-s^{\prime}\right)\|f\|_{\infty} \\
&=\left(\frac{\left|e^{\left(s-s^{\prime}\right) z}-1\right|}{|\operatorname{Re} z|}+\left(s-s^{\prime}\right)\right)\|f\|_{\infty} \\
& \rightarrow 0
\end{aligned}
$$

as $s-s^{\prime} \rightarrow 0$.

For $f$ in $B U C\left(\mathbb{R}_{+}, X\right)$ with singular set $i E$, let $g: V \rightarrow X$ be a holomorphic extension of $\tilde{f}$ to a connected open set $V$ containing $\mathbb{C}_{+} \cup i(\mathbb{R} \backslash E)$. Propositions 2.1 and 3.1 show that we may define $G: V \rightarrow B U C\left(\mathbb{R}_{+}, X\right)$ by

$$
G(z)(s)=g_{s}(z) \quad\left(s \in \mathbb{R}_{+}, z \in V\right) .
$$

Proposition 3.2. Let $f: \mathbb{R}_{+} \rightarrow X$ be bounded and uniformly continuous, and let $G: V \rightarrow B U C\left(\mathbb{R}_{+}, X\right)$ be as above. Then $G$ is holomorphic. 
Proof. Let $\lambda \in V$, and let $r$ and $c$ be as in Proposition 2.1. For $\lambda^{\prime} \in B(\lambda, r)$,

$$
\begin{aligned}
\left\|G(\lambda)(s)-G\left(\lambda^{\prime}\right)(s)\right\| & =\left\|\frac{1}{2 \pi i} \int_{|z-\lambda|=r}\left(\frac{g_{s}(z)}{z-\lambda}-\frac{g_{s}(z)}{z-\lambda^{\prime}}\right) d z\right\| \\
& \leq r \sup _{|z-\lambda| \leq r}\left\|g_{s}(z)\right\| \sup _{|z-\lambda|=r}\left|\frac{1}{z-\lambda}-\frac{1}{z-\lambda^{\prime}}\right| \\
& \leq c \frac{\left|\lambda-\lambda^{\prime}\right|}{r-\left|\lambda-\lambda^{\prime}\right|} \\
& \rightarrow 0 \quad \text { as } \lambda^{\prime} \rightarrow \lambda,
\end{aligned}
$$

uniformly in $s$. Thus $G: V \rightarrow B U C\left(\mathbb{R}_{+}, X\right)$ is continuous.

Let $\gamma$ be a closed contour in $V$. Then

$$
\left(\int_{\gamma} G(z) d z\right)(s)=\int_{\gamma} g_{s}(z) d z=0 .
$$

By Morera's Theorem, $G$ is holomorphic.

Proposition 3.3. Let $f: \mathbb{R}_{+} \rightarrow X$ be bounded and uniformly continuous. Then $\sigma_{u}(D, f)$ is the set of all singular points of $\tilde{f}$ in $i \mathbb{R}$.

Proof. Suppose that $\lambda$ in $i \mathbb{R}$ is a regular point of $R(\cdot, D) f$, and let $G$ be a holomorphic extension of this function near $\lambda$. Let $g(z)=G(z)(0)$. For Re $z>0$,

$$
g(z)=(R(z, D) f)(0)=\int_{0}^{\infty} e^{-t z}(S(t) f)(0) d t=\int_{0}^{\infty} e^{-t z} f(t) d t=\widetilde{f}(z) .
$$

Thus $g$ is a holomorphic extension of $\tilde{f}$, so $\lambda$ is a regular point of $\tilde{f}$.

Suppose that $\lambda$ in $i \mathbb{R}$ is a regular point of $\widetilde{f}$. Let $g: V \rightarrow X$ be a holomorphic extension of $\widetilde{f}$ near $\lambda$. By Proposition 3.2, there is a holomorphic function $G: V \rightarrow$ $B U C\left(\mathbb{R}_{+}, X\right)$ such that, for $\operatorname{Re} z>0$ and $s \geq 0$,

$$
G(z)(s)=g_{s}(z)=\int_{0}^{\infty} e^{-t z} f(s+t) d t=\int_{0}^{\infty} e^{-t z}(S(t) f)(s) d t=(R(z, D) f)(s) .
$$

Thus $G$ extends $R(\cdot, D) f$, so $\lambda \notin \sigma_{u}(D, f)$.

The following example shows that the local unitary spectrum at $x$ of the generator of a (bounded) semigroup may be much smaller than the imaginary part of the spectrum of the generator restricted to any closed invariant subspace containing $x$. This contrasts with the situation for bounded $C_{0}$-groups, where the local unitary spectrum (now defined by means of the resolvent on $\mathbb{C} \backslash i \mathbb{R}$ instead of $\mathbb{C}_{+}$) coincides with the spectrum of the generator on a subspace [29, Propositions 3.4, 3.5].

Example 3.4. Let $X=\mathbb{C}$. There is a function $f$ in $C_{0}\left(\mathbb{R}_{+}\right)$such that the linear span of $\left\{f_{t}: t \geq 0\right\}$ is dense in $C_{0}\left(\mathbb{R}_{+}\right)$, but $\widetilde{f}(z)$ extends to a holomorphic function for Re $z>-1$. By Proposition 3.3, $\sigma_{u}(D, f)$ is empty, but the spectrum of the generator of the translation semigroup $\mathbf{S}$ on the closed linear span of the orbit of $f$ under $\mathbf{S}$ is the closed left half-plane. 
To construct such an $f$, let $\left\{f^{(k)}: k=1,2, \ldots\right\}$ be a dense subset of the unit ball of $\left\{g \in C_{0}\left(\mathbb{R}_{+}\right): g(0)=0\right\}$ such that each $f^{(k)}$ has support in $\left[0,2^{k}\right]$, and let

$$
\begin{aligned}
f(t)=\exp \left(-\left(2^{n+1}+2^{k+1}\right)^{2}\right) f^{(k)}\left(t-2^{n+1}-2^{k}\right) \\
\\
\left(2^{n+1}+2^{k} \leq t<2^{n+1}+2^{k+1} ; k=1, \ldots, n ; n=1,2, \ldots\right),
\end{aligned}
$$

with $f(t)=0$ for all remaining $t \geq 0$. Then

$$
\widetilde{f}(z)=\sum_{n=1}^{\infty} \sum_{k=1}^{n} \exp \left(-\left(2^{n+1}+2^{k}\right) z-\left(2^{n+1}+2^{k+1}\right)^{2}\right)\left(f^{(k)}\right) \widetilde{r}(z) .
$$

This series converges locally uniformly to a holomorphic function on $\mathbb{C}$, and the closed linear span of the translates of $f$ contains each $f^{(k)}$.

\section{The Tauberian theorems}

We are now in position to give the Tauberian theorems, starting with the case of bounded, uniformly continuous functions.

Recall that a bounded, uniformly continuous function $h: \mathbb{R} \rightarrow X$ is said to be almost periodic if $h$ can be approximated uniformly by linear combinations of functions of the form $t \mapsto e^{i a t} x(a \in \mathbb{R}, x \in X)$. A function $f: \mathbb{R}_{+} \rightarrow X$ is said to be asymptotically almost periodic if there is an almost periodic function $h: \mathbb{R} \rightarrow X$ such that $\|f(t)-h(t)\| \rightarrow 0$ as $t \rightarrow \infty$.

Theorem 4.1. Let $f: \mathbb{R}_{+} \rightarrow X$ be bounded and uniformly continuous, and suppose that the singular set $i E$ of $\tilde{f}$ in $i \mathbb{R}$ is countable. Suppose also that, for each $\eta$ in $E$,

$$
\lim _{\alpha \searrow 0} \alpha \tilde{f}_{s}(\alpha+i \eta)
$$

exists, uniformly for $s \geq 0$. Then $f$ is asymptotically almost periodic.

If the limit in $\left(^{*}\right)$ is 0 , uniformly in $s$, for each $\eta$ in $E$, then $\|f(t)\| \rightarrow 0$ as $t \rightarrow \infty$.

Proof. By Proposition 3.3, $\sigma_{u}(D, f)=i E$. Condition $(*)$ implies that

$$
\lim _{\alpha \searrow 0} \alpha \int_{0}^{\infty} e^{-(\alpha+i \eta) t} S(t) f d t=\lim _{\alpha \searrow 0} \alpha R(\alpha+i \eta, D) f
$$

exists in $B U C\left(\mathbb{R}_{+}, X\right)$. By [9, Theorem 6.1], $\{S(t) f: t \geq 0\}$ is relatively compact. By the Glicksberg-de Leeuw Theorem [22, Section 2.4], applied in the closed linear span $Y$ of $\left\{f_{t}: t \geq 0\right\}, f=g+h$, where $g, h \in Y,\|S(t) g\| \rightarrow 0$ and $h$ is in the closed linear span of the unimodular eigenvectors of $\mathbf{S}$, so $h$ extends to an almost periodic function on $\mathbb{R}$.

If the limits in $\left(^{*}\right)$ are all 0 , then $h=0$, by [9, Theorem 3.4].

For the real line $\mathbb{R}$, a related result is proved in [25, Theorem 3.9].

Remark. In Theorem 4.1, it suffices that the limits in $\left(^{*}\right)$ exist uniformly for $s$ in some subset $Q$ of $\mathbb{R}_{+}$, where $Q$ has the property that there is a constant $d$ such that each point of $\mathbb{R}_{+}$is within distance $d$ of $Q$ (for example, $Q=\mathbb{Z}_{+}$). This follows from the formula

$$
\widetilde{f}_{s}(z)=e^{\left(s-s^{\prime}\right) z} \widetilde{f}_{s^{\prime}}(z)-\int_{s^{\prime}}^{s} e^{(s-t) z} f(t) d t
$$


Now we give an example which shows (in the case $\beta=0$ ) that the assumption of uniform convergence in $s$ cannot be omitted from Theorem 4.1, and which also exhibits (in the case $0<\beta<1 / 2$ ) the difference between Theorem 4.1 and [1, Theorem 4.3].

Example 4.2. Let $X=\mathbb{C}$ and $f(t)=t^{-\beta} \sin \sqrt{t}$, where $0 \leq \beta<1 / 2$. Then $f$ is bounded and uniformly continuous. By expanding $\sin \sqrt{t}$ as a power series in $\sqrt{t}$ and integrating term by term,

$$
\widetilde{f}(z)=\frac{1}{z^{\frac{3}{2}-\beta}} \sum_{n=0}^{\infty} \frac{(-1)^{n+1} \Gamma\left(n+\frac{3}{2}-\beta\right)}{(2 n+1) ! z^{n}} \quad(\operatorname{Re} z>0) .
$$

This function has a holomorphic extension to $\mathbb{C} \backslash(-\infty, 0]$, so the singular set of $\tilde{f}$ is $\{0\}$.

In the case $\beta=0$,

$$
\begin{gathered}
\widetilde{f}(z)=\frac{\sqrt{\pi} e^{-1 / 4 z}}{2 z^{3 / 2}}, \\
\alpha \widetilde{f}_{s}(\alpha)=\alpha e^{s \alpha}\left(\frac{\sqrt{\pi} e^{-1 / 4 \alpha}}{2 \alpha^{3 / 2}}-\int_{0}^{s} e^{-\alpha t} \sin \sqrt{t} d t\right) \rightarrow 0
\end{gathered}
$$

as $\alpha \searrow 0$, for each $s \geq 0$. Nevertheless, $f(t)$ does not tend to 0 . See [26, p. 608] and [25, Example 3.12] for other treatments of this example.

In the case $0 \leq \beta<1 / 2$,

$$
\int_{\left(\left(2 n+\frac{1}{4}\right) \pi\right)^{2}}^{\left(\left(2 n+\frac{3}{4}\right) \pi\right)^{2}} f(t) d t \geq \frac{(4 n+1) \pi^{2}}{2 \sqrt{2}\left(\left(2 n+\frac{3}{4}\right) \pi\right)^{\frac{\beta}{2}}} \rightarrow \infty
$$

as $n \rightarrow \infty$. Hence $\sup _{t \geq 0}\left|\int_{0}^{t} f(s) d s\right|=\infty$. Thus $f$ does not satisfy the conditions of [1, Theorem 4.1]. But for $0<\beta<1 / 2, f$ does satisfy the conditions of Theorem 4.1 and Theorem 4.3 of this paper.

Next, we give two versions of Theorem 4.1 in which we assume either that $f$ is bounded or that it is uniformly continuous. Theorem 4.3 should be compared with [2, Theorem 4.1], and Corollary 4.4 with [5, Theorem 2.3], [10, Theorem 1] and [3, Theorem 3.5].

Theorem 4.3. Let $f: \mathbb{R}_{+} \rightarrow X$ be a bounded measurable function, and suppose that the singular set $i E$ of $\widetilde{f}$ in $i \mathbb{R}$ is countable. Suppose also that, for each $\eta$ in $E$,

$$
\lim _{\alpha \searrow 0} \alpha \widetilde{f}_{s}(\alpha+i \eta)=0
$$

uniformly for $s \geq 0$. Then $\int_{0}^{t} e^{-i \mu u} f(u) d u \rightarrow \widetilde{f}(i \mu)$ as $t \rightarrow \infty$, for each $\mu$ in $\mathbb{R} \backslash E$, where $\tilde{f}$ is considered to be extended holomorphically near $i \mu$.

Proof. Replacing $f(t)$ by $e^{-i \mu t} f(t)$, we may first assume that $\mu=0$. Then replacing $f(t)$ by $f(t)-e^{-t} \widetilde{f}(0)$, we may assume that $\widetilde{f}(0)=0$.

Let

$$
g(t)=\int_{0}^{t} f(u) d u=\widetilde{f}(0)-\widetilde{f}_{t}(0) .
$$


Then $g$ is uniformly continuous, since $f$ is bounded, and $g$ is bounded, by Proposition 2.1. For $\operatorname{Re} z>0, \widetilde{g}(z)=\widetilde{f}(z) / z$, whose singularity at $z=0$ is removable, so $\widetilde{g}$ has singular set $i E$. Moreover,

$$
\widetilde{g}_{s}(z)=\frac{\widetilde{f}_{s}(z)+g(s)}{z} \quad(\operatorname{Re} z>0)
$$

so, for $\eta$ in $E$,

$$
\lim _{\alpha \searrow 0} \alpha \widetilde{g}_{s}(\alpha+i \eta)=0
$$

uniformly in $s$, since $g$ is bounded and $\left(^{*}\right)$ holds. We may therefore apply Theorem 4.1 to $g$, and deduce that $g(t) \rightarrow 0$ as $t \rightarrow \infty$.

Remarks. 1. The last statement of Theorem 4.1 can be recovered from Theorem 4.3, since convergence of $\int_{0}^{t} e^{-i \mu u} f(u) d u($ as $t \rightarrow \infty)$ for some $\mu$ in $\mathbb{R}$ implies convergence of $f(t)$ to 0 , if $f$ is uniformly continuous.

2. For bounded functions, Abel convergence is equivalent to Cesàro convergence [15, Theorem 18.3.3]. The corresponding statement for convergence uniformly in $s$ also holds. Thus the limits in $\left({ }^{*}\right)$ of Theorems 4.1 and 4.3 may be replaced by

$$
\lim _{t \rightarrow \infty} \frac{1}{t} \int_{0}^{t} e^{-i \eta u} f(s+u) d u \text {. }
$$

Corollary 4.4. Let $f: \mathbb{R}_{+} \rightarrow X$ be uniformly continuous, and suppose that the singular set $i E$ of $\tilde{f}$ in $i \mathbb{R}$ is countable. Suppose also that, for each $\eta$ in $E$,

$$
\lim _{\alpha \searrow 0} \alpha \widetilde{f}_{s}(\alpha+i \eta)=0
$$

uniformly for $s \geq 0$. Then $\|f(t)\| \rightarrow 0$ as $t \rightarrow \infty$.

Proof. We follow the argument of [5, Theorem 2.3], [10, Theorem 1] and [3, Theorem 3.5]. Take $\delta>0$, and let

$$
g(t)=f(t+\delta)-f(t) .
$$

Then $g$ is bounded and uniformly continuous. For $\operatorname{Re} z>0$,

$$
\widetilde{g}(z)=\int_{0}^{\infty} e^{-t z} f(t+\delta) d t-\widetilde{f}(z)=\left(e^{\delta z}-1\right) \widetilde{f}(z)-e^{\delta z} \int_{0}^{\delta} e^{-t z} f(t) d t .
$$

Thus the singular set of $g$ is contained in $i E$. For $\eta$ in $E$,

$$
\lim _{\alpha \searrow 0} \alpha \widetilde{g}_{s}(\alpha+i \eta)=\lim _{\alpha \searrow 0} \alpha \widetilde{f}_{s+\delta}(\alpha+i \eta)-\lim _{\alpha \searrow 0} \alpha \widetilde{f}_{s}(\alpha+i \eta)=0
$$

uniformly in $s$. If $0 \notin E$, then Theorem 4.3 can be applied with $\mu=0$, giving

$$
0=\lim _{t \rightarrow \infty}\left\|\int_{0}^{t} g(u) d u-\widetilde{g}(0)\right\|=\lim _{t \rightarrow \infty}\left\|\int_{t}^{t+\delta} f(u) d u\right\| .
$$

Since this holds for all $\delta>0$ and since $f$ is uniformly continuous, it follows that $\|f(t)\| \rightarrow 0$, if $0 \notin E$.

Now, suppose that $0 \in E$. By hypothesis, there exists $\alpha>0$ such that $\left\|\alpha \widetilde{f}_{s}(\alpha)\right\|$ $<1$ for all $s \geq 0$. By uniform continuity, there exists $\kappa$ such that $\|f(s+t)-f(s)\|$ $<\kappa(1+t)$ for all $s \geq 0$ and $t \geq 0$. Now

$$
\|f(s)\|=\left\|\alpha \widetilde{f}_{s}(\alpha)-\int_{0}^{\infty} \alpha e^{-\alpha t}(f(s+t)-f(s)) d t\right\|<1+\kappa+\kappa / \alpha .
$$


Thus $f$ is bounded, so the result follows from Theorem 4.1.

Corollary 4.5. Let $f: \mathbb{R}_{+} \rightarrow X$ be bounded and uniformly continuous, and suppose that the singular set of $\tilde{f}$ is contained in $2 \pi \omega^{-1} i \mathbb{Z}$ for some $\omega>0$. Then $\|f(t+\omega)-f(t)\| \rightarrow 0$ as $t \rightarrow \infty$.

Proof. Let $g(t)=f(t+\omega)-f(t)$. For $s \geq 0, n \in \mathbb{Z}$, and $\alpha>0$, a calculation similar to Corollary 4.4 shows that

$$
\begin{aligned}
\| \alpha \widetilde{g}_{s}(\alpha & +2 \pi i n / \omega) \| \\
& =\left\|\alpha\left(e^{\alpha \omega}-1\right) \widetilde{f}_{s}(\alpha+2 \pi i n / \omega)-\alpha e^{\alpha \omega} \int_{0}^{\omega} e^{-(\alpha+2 \pi i n / \omega) u} f(s+u) d u\right\| \\
& \leq\left(e^{\alpha \omega}-1\right)\|f\|_{\infty}+\alpha e^{\alpha \omega} \omega\|f\|_{\infty} \\
& \rightarrow 0
\end{aligned}
$$

as $\alpha \searrow 0$, uniformly in $s$. Thus the result follows from Corollary 4.4.

To conclude this section, we apply other results from [9] to the classical situation of Laplace transforms.

Theorem 4.6. Let $f: \mathbb{R}_{+} \rightarrow X$ be bounded and uniformly continuous, and suppose that the singular set of $\tilde{f}$ in $i \mathbb{R}$ is countable. Let $Y$ be the closed linear span of $\left\{f_{t}: t \geq 0\right\}$ in $B U C\left(\mathbb{R}_{+}, X\right)$. For any $\alpha>\limsup _{t \rightarrow \infty}\|f(t)\|$, there exists $g$ in $Y$ such that $\|f-g\|_{\infty}<\alpha$ and $g(t) \rightarrow 0$ as $t \rightarrow \infty$.

Proof. This follows from Proposition 3.3, [9, Theorem 7.4] and the observation that the local spectrum of $D$ at $f$ is unchanged by restriction to $Y$ (see the remark after Theorem 2.2 of $[9])$.

Recall that a function $g$ in $L^{1}(\mathbb{R})$ is said to be of spectral synthesis with respect to a closed subset $E$ of $\mathbb{R}$ if there is a sequence $\left(g_{n}\right)$ in $L^{1}(\mathbb{R})$ such that $\left\|g_{n}-g\right\|_{1} \rightarrow 0$ and, for each $n$, the Fourier transform $\widehat{g}_{n}$ vanishes on some neighbourhood of $E$ in $\mathbb{R}$. For $g$ in $L^{1}\left(\mathbb{R}_{+}\right)$, we consider $g$ to be extended to $\mathbb{R}$ by putting $g(s)=0$ for $s<0$, so it is meaningful to talk of $g$ being of spectral synthesis.

The next result is a local version of [14, Théorème II.7].

Theorem 4.7. Let $f: \mathbb{R}_{+} \rightarrow X$ be bounded and strongly measurable, and let $i E$ be the singular set of $\tilde{f}$ in $i \mathbb{R}$. Let $g \in L^{1}\left(\mathbb{R}_{+}\right)$and suppose that $g$ is of spectral synthesis with respect to $-E$. Then

$$
\left\|\int_{0}^{\infty} g(s) f(s+t) d s\right\| \rightarrow 0
$$

as $t \rightarrow \infty$.

Proof. If $f$ is bounded and uniformly continuous, this result follows from Proposition 3.3 and [9, Theorem 5.1]. We shall use this special case to obtain the general result.

Suppose that $f$ is bounded and strongly measurable. Let

$$
f_{n}(t)=n \int_{0}^{1 / n} f(t+u) d u
$$


Then $f_{n}$ is bounded and uniformly continuous. For Re $z>0$,

$$
\begin{aligned}
\tilde{f}_{n}(z) & =n \int_{0}^{\infty} \int_{0}^{1 / n} e^{-t z} f(t+u) d u d t \\
& =n \int_{0}^{1 / n} \int_{0}^{\infty} e^{-(t+u) z} f(t+u) d t e^{u z} d u \\
& =n \int_{0}^{1 / n} \int_{u}^{\infty} e^{-t z} f(t) d t e^{u z} d u \\
& =n \int_{0}^{1 / n} \widetilde{f}(z) e^{u z} d u-n \int_{0}^{1 / n} \int_{0}^{u} e^{-(t-u) z} f(t) d t d u \\
& =n\left(\frac{e^{z / n}-1}{z}\right) \widetilde{f}(z)-n \int_{0}^{1 / n} \int_{0}^{u} e^{-(t-u)} f(t) d t d u .
\end{aligned}
$$

Thus the singular set of $\widetilde{f}_{n}$ is contained in $i E$. It follows from the first paragraph that

$$
\left\|\int_{0}^{\infty} g(s) f_{n}(s+t) d s\right\| \rightarrow 0 \quad \text { as } t \rightarrow \infty
$$

But

$$
\begin{aligned}
\| \int_{0}^{\infty} g(s) f_{n}(s+t) & d s-\int_{0}^{\infty} g(s) f(s+t) d s \| \\
= & \left\|n \int_{0}^{1 / n} \int_{0}^{\infty} g(s) f(s+t+u) d s d u-\int_{0}^{\infty} g(s) f(s+t) d s\right\| \\
& =\left\|n \int_{0}^{1 / n} \int_{0}^{\infty}(g(s-u)-g(s)) f(s+t) d s d u\right\| \\
& \leq\|f\|_{\infty} \sup _{0 \leq u \leq 1 / n} \int_{0}^{\infty}|g(s-u)-g(s)| d s \\
& \rightarrow 0 \text { as } n \rightarrow \infty \text { (uniformly in } t),
\end{aligned}
$$

where we make the convention that $g(s)=0$ if $s<0$. It follows that

$$
\left\|\int_{0}^{\infty} g(s) f(s+t) d s\right\| \rightarrow 0
$$

Corollary 4.8. Let $f: \mathbb{R}_{+} \rightarrow X$ be bounded and strongly measurable, and let $i E$ be the singular set of $\tilde{f}$ in $i \mathbb{R}$. Let $g: \mathbb{R}_{+} \rightarrow \mathbb{C}$ be measurable, and suppose that $\int_{0}^{\infty}\left(1+t^{\alpha}\right)|g(t)| d t<\infty$ for some $\alpha>1 / 2$ and that $\int_{0}^{\infty} e^{i \eta t} g(t) d t=0$ for all $\eta$ in E. Then

$$
\left\|\int_{0}^{\infty} g(s) f(s+t) d s\right\| \rightarrow 0
$$

as $t \rightarrow \infty$.

Proof. This follows from Theorem 4.7 and the fact that $g$ is of spectral synthesis with respect to $-E[24$, Theorem 11.1]. 


\section{The Cauchy problem}

Consider an abstract inhomogeneous Cauchy problem

$$
u^{\prime}(t)=A u(t)+f(t) \quad(t \geq 0), \quad u(0)=x .
$$

We assume throughout this section that $A$ is a closed linear operator on a Banach space $X, \lambda I-A$ is injective for all sufficiently large real $\lambda, x \in X$ and $f \in B U C\left(\mathbb{R}_{+}, X\right)$. We seek to obtain information about $u$ from assumptions about $A$ and $f$. Similar problems have been considered in [4], [12] (homogeneous problems) and [25] (problems on $\mathbb{R})$.

A mild solution of (ICP) is a continuous function $u: \mathbb{R}_{+} \rightarrow X$ such that if $v(t)=\int_{0}^{t} u(s) d s$, then $v(t) \in D(A)$ and $v^{\prime}(t)=A v(t)+x+\int_{0}^{t} f(s) d s$ for all $t \geq 0$.

Proposition 5.1. Let $u$ be a bounded, uniformly continuous, mild solution of the inhomogeneous Cauchy problem (ICP).

1. For $\operatorname{Re} z>0, \widetilde{u}(z) \in D(A)$ and $(z I-A) \widetilde{u}(z)=x+\widetilde{f}(z)$.

2. Suppose that there is a holomorphic function $g: \mathbb{C}_{+} \rightarrow X$ such that $g(z) \in$ $D(A)$ and $(z I-A) g(z)=x+\widetilde{f}(z)$ for all $z$ in $\mathbb{C}_{+}$. Then $g(z)=\widetilde{u}(z)$ for all $z$ in $\mathbb{C}_{+}$.

Proof. (1). For Re $z>0$,

$$
\int_{0}^{\infty} A v(t) e^{-t z} d t=\int_{0}^{\infty}\left(u(t)-x-\int_{0}^{t} f(s) d s\right) e^{-t z} d t=\widetilde{u}(z)-z^{-1} x-z^{-1} \widetilde{f}(z) .
$$

Since these integrals are absolutely convergent and $A$ is closed, $\widetilde{v}(z) \in D(A)$ and

$$
A \widetilde{v}(z)=\widetilde{u}(z)-z^{-1} x-z^{-1} \widetilde{f}(z) .
$$

Now, $\widetilde{u}(z)=z \widetilde{v}(z)$, so $\widetilde{u}(z) \in D(A)$ and

$$
A \widetilde{u}(z)=z \widetilde{u}(z)-x-\widetilde{f}(z) .
$$

(2). For $\operatorname{Re} z>0$,

$$
(z I-A) g(z)=x+\widetilde{f}(z)=(z I-A) \widetilde{u}(z) .
$$

For all sufficiently large real $\lambda, \lambda I-A$ is injective, so $g(\lambda)=\widetilde{u}(\lambda)$. It follows by analytic continuation that $g(z)=\widetilde{u}(z)$ whenever $\operatorname{Re} z>0$.

Corollary 5.2. Let $u$ be a bounded, uniformly continuous, mild solution of (ICP). The singular set of $\widetilde{u}$ in $i \mathbb{R}$ is the set of all points $\lambda$ in $i \mathbb{R}$ such that there is no holomorphic function $g: U \rightarrow X$, defined on an open set $U$ containing $\mathbb{C}_{+} \cup\{\lambda\}$, with $g(z) \in D(A)$ and $(z I-A) g(z)=x+\widetilde{f}(z)$ for all $z$ in $\mathbb{C}_{+}$.

Theorem 4.1 now has the following formulation for solutions of (ICP). Other sufficient conditions for the solutions to be asymptotically almost periodic have been given in [4]. In the case of solutions defined on the whole line $\mathbb{R}$, a sharper result has been given in [25, Theorem 4.3].

Theorem 5.3. Let u be a bounded, uniformly continuous, mild solution of (ICP). Suppose that there is a subset $E$ of $\mathbb{R}$ such that

1. E is countable, 
2. there is an open set $U$ containing $\mathbb{C}_{+} \cup i(\mathbb{R} \backslash E)$ and a holomorphic function $g: U \rightarrow X$ such that $g(z) \in D(A)$ and $(z I-A) g(z)=x+\widetilde{f}(z)$ for all $z$ in $\mathbb{C}_{+}$,

3. for each $\eta$ in $E$,

$$
\lim _{\alpha \searrow 0} \alpha \widetilde{u}_{s}(\alpha+i \eta)
$$

exists, uniformly for $s \geq 0$.

Then $u$ is asymptotically almost periodic. If the limits in 3 are all zero, then $\|u(t)\| \rightarrow 0$ as $t \rightarrow \infty$.

When the conditions of Theorem 5.3 are satisfied, $u=u_{1}+u_{2}$, where $\left\|u_{1}(t)\right\| \rightarrow$ $0, u_{2}$ extends to an almost periodic function on $\mathbb{R}$, and $u_{1}$ and $u_{2}$ belong to the closed linear span of the translates of $u$.

In the homogeneous case when $f=0, u_{1}$ and $u_{2}$ are mild solutions of the homogeneous Cauchy problem and $u_{2}$ can be approximated uniformly by linear combinations of functions of the form $t \mapsto e^{i \mu t} y$ where $\mu \in \mathbb{R}, y \in D(A)$ and $A y=i \mu y$. In this case, the following result shows that condition (2) of Theorem 5.3 is automatically satisfied when $E$ contains $\sigma_{a p}(A) \cap i \mathbb{R}$, where $\sigma_{a p}(A)$ is the approximate point spectrum of $A$. Hence Theorem 5.3 extends [12, Theorem 4].

Proposition 5.4. Let $u$ be a bounded, uniformly continuous, mild solution of the homogeneous Cauchy problem. The singular set of $\widetilde{u}$ in $i \mathbb{R}$ is contained in the approximate point spectrum $\sigma_{a p}(A)$ of $A$.

Proof. Let $Z_{0}$ be the Hille-Yosida space [18], [21], consisting of all those $y$ in $X$ for which there is a bounded, uniformly continuous, mild solution $v(t, y)$ of the homogeneous Cauchy problem with $v(0, y)=y$. In the norm

$$
\|y\|_{z_{0}}=\sup _{t \geq 0}\|v(t, y)\|
$$

$Z_{0}$ becomes a Banach space which is isometrically isomorphic to a closed subspace $Y$ of $B U C\left(\mathbb{R}_{+}, X\right)$ under the map $V$ given by

$$
(V y)(t)=v(t, y)
$$

Moreover, $Y$ is invariant under the translation semigroup $\mathbf{S}$, and the corresponding $C_{0}$-semigroup $\mathbf{T}_{0}$ on $Z_{0}$ is given by

$$
T_{0}(t) y=v(t, y)
$$

The generator $A_{0}$ of $\mathbf{T}_{0}$ is the part of $A$ in $Z_{0}$ and $\sigma\left(A_{0}\right) \subseteq \sigma(A)$ [11, Theorem 5.5], [12, Corollary 2].

For $\operatorname{Re} z>0, \widetilde{u}(z)=R\left(z, A_{0}\right) x$. Hence any singular point $\lambda=i \eta$ is in the topological boundary of $\sigma\left(A_{0}\right)$, and hence in the approximate point spectrum of $A_{0}$. Thus there is a sequence $\left(x_{n}\right)$ in $D\left(A_{0}\right)$ such that $\left\|x_{n}\right\|_{Z_{0}}=1$ and $\left\|A_{0} x_{n}-\lambda x_{n}\right\|_{Z_{0}} \rightarrow$ 0 . There exist $t_{n} \geq 0$ such that $\left\|T_{0}\left(t_{n}\right) x_{n}\right\| \geq 1 / 2$. If we put $y_{n}=T_{0}\left(t_{n}\right) x_{n}$, then $\left\|y_{n}\right\| \geq 1 / 2, y_{n} \in \bar{D}(A)$ and

$$
\left\|A y_{n}-\lambda y_{n}\right\| \leq\left\|A_{0} y_{n}-\lambda y_{n}\right\|_{z_{0}} \leq\left\|A_{0} x_{n}-\lambda x_{n}\right\|_{z_{0}} \rightarrow 0 .
$$

Thus $\lambda$ is an approximate eigenvalue of $A$.

Now, suppose that the homogeneous Cauchy problem is well-posed, so that $A$ generates a $C_{0}$-semigroup $\mathbf{T}=\{T(t): t \geq 0\}$ on $X$. Then the assumptions of this section are satisfied, and mild solutions of the Cauchy problem are of the 
form $u(t, x)=T(t) x$. For a bounded mild solution $u(t, x)$, the singular set of $\widetilde{u}$ is the local unitary spectrum $\sigma_{u}(A, x)$. Thus Theorem 5.3 extends [9, Theorem 6.1] and [9, Theorem 3.4] from orbits of bounded semigroups to bounded, uniformly continuous orbits of general semigroups, and Theorem 4.7 permits [9, Theorem 5.1] to be extended in the same way. We can also extend part of [9, Theorem 7.2], as follows.

Theorem 5.5. Let $\mathbf{T}$ be a trivially asymptotically stable $C_{0}$-semigroup on $X$ with generator $A$ (so that $\lim \sup _{t \rightarrow \infty}\|T(t) y\|>0$ for all non-zero $y$ in $X$ ). Let $x \in X$, and $u(t)=T(t) x$. Suppose that $u$ is bounded and uniformly continuous, and that $\sigma_{u}(A, x)$ is countable. Then $\mathbf{T}$ has a complete bounded orbit through $x$, that is, there is a bounded family $\left\{x_{t} ; t \in \mathbb{R}\right\}$ in $X$ such that $x_{0}=x$ and $T(s) x_{t}=x_{s+t}(t \in$ $\left.\mathbb{R}, s \in \mathbb{R}_{+}\right)$.

Proof. Consider the shift semigroup $\mathbf{S}$ restricted to the closed subspace $Y$ of $B U C\left(\mathbb{R}_{+}, X\right)$ generated by $\left\{u_{t}: t \geq 0\right\}$. This subspace consists of orbits of $\mathbf{T}$, so the shift semigroup is trivially asymptotically stable. By Proposition 3.3 and $\left[9\right.$, Theorem 7.2], $\mathbf{S}$ has a complete isometric orbit $\left\{v_{t}: t \in \mathbb{R}\right\}$ through $u$ in $Y$. Putting $x_{t}=v_{t}(0)$ produces the required complete bounded orbit in $X$.

Examples of unbounded semigroups where results of this type are applicable can be constructed in a similar way to Example 3.4, but working in weighted spaces such as

$$
X=\left\{f \in C_{0}\left(\mathbb{R}_{+}\right):\|f(t)\| /(1+t) \rightarrow 0 \text { as } t \rightarrow \infty\right\} .
$$

\section{The Discrete CASE}

In this section, we adapt our results to the case of power series. As in [5, Section $5]$, we deduce them from those about Laplace transforms by introducing an auxiliary function $f$. In the present context, we need to arrange that $f$ is both bounded and uniformly continuous.

Suppose that $\left(a_{n}\right)_{n \geq 0}$ is a sequence in $X$ such that $\limsup _{n \rightarrow \infty}\left\|a_{n}\right\|^{1 / n} \leq 1$. Let $h(z)$ be the power series

$$
h(z)=\sum_{n=0}^{\infty} a_{n} z^{n} \quad(|z|<1) .
$$

Let $E$ be the singular set of $h$ in the unit circle $\Gamma$. For $m \geq 0$, let

$$
h_{m}(z)=\sum_{n=0}^{\infty} a_{n+m} z^{n}=\frac{h(z)-\sum_{n=0}^{m-1} a_{n} z^{n}}{z^{m}} \quad(0<|z|<1) .
$$

Define $f: \mathbb{R}_{+} \rightarrow X$ by

$$
f(t)=(n+1-t) a_{n}+(t-n) a_{n+1} \quad(n \leq t<n+1 ; n \geq 0) .
$$


For $\operatorname{Re} z>0$,

$$
\begin{aligned}
\widetilde{f}(z) & =\sum_{n=0}^{\infty} \int_{n}^{n+1}\left((n+1-t) a_{n}+(t-n) a_{n+1}\right) e^{-t z} d t \\
& =\sum_{n=0}^{\infty} a_{n} e^{-n z}\left(\int_{0}^{1}(1-t) e^{-t z} d t\right)+\sum_{n=0}^{\infty} a_{n+1} e^{-(n+1) z} e^{z}\left(\int_{0}^{1} t e^{-t z} d t\right) \\
& =h\left(e^{-z}\right)\left(\frac{z+e^{-z}-1}{z^{2}}\right)+\left(h\left(e^{-z}\right)-a_{0}\right)\left(\frac{e^{z}-z-1}{z^{2}}\right) \\
& =h\left(e^{-z}\right)\left(\frac{2(\cosh z-1)}{z^{2}}\right)-a_{0}\left(\frac{e^{z}-1-z}{z^{2}}\right) .
\end{aligned}
$$

Thus the singular set $i E^{\prime}$ of $\tilde{f}$ in $i \mathbb{R}$ is contained in $\left\{i \eta: \eta \in \mathbb{R}, e^{-i \eta} \in E\right\}$. Moreover, for $\eta$ in $E^{\prime}$ and $m \geq 0$,

$$
\lim _{\alpha \searrow 0} \alpha \widetilde{f}_{m}(\alpha+i \eta)=\left(\lim _{r \nearrow 1}(1-r) h_{m}\left(r e^{-i \eta}\right)\right)\left(\frac{2(1-\cos \eta)}{\eta^{2}}\right),
$$

since $\left(1-e^{-\alpha}\right) / \alpha \rightarrow 1$ as $\alpha \searrow 0$.

This enables us to deduce the following results from those in Section 4.

Theorem 6.1. Let $\left(a_{n}\right)_{n \geq 0}$ be a bounded sequence in $X$, and let $h(z)=\sum_{n=0}^{\infty} a_{n} z^{n}$ for $|z|<1$. Suppose that the singular set $E$ of $h$ in the unit circle $\Gamma$ is countable, and that, for each $\lambda$ in $E$,

$$
\lim _{r \nearrow 1}(1-r) h_{m}(r \lambda)
$$

exists, uniformly for $m \geq 0$. Then there is an almost periodic sequence $\left(b_{n}\right)_{n \in \mathbb{Z}}$ in $X$ such that $\left\|a_{n}-b_{n}\right\| \rightarrow 0$ as $n \rightarrow \infty$.

Proof. This follows from Theorem 4.1 and the subsequent remark.

The following Tauberian theorem for power series is more general than $[1$, Theorem $\left.2^{\prime}\right]$ and may be compared with [1, Theorem 4].

Theorem 6.2. Let $\left(a_{n}\right)_{n \geq 0}$ be a sequence in $X$ such that $\sup _{n}\left\|a_{n}-a_{n+1}\right\|<\infty$, and let $h(z)=\sum_{n=0}^{\infty} a_{n} z^{n}$ for $|z|<1$. Suppose that the singular set $E$ of $h$ in the unit circle $\Gamma$ is countable, $1 \notin E$, and, for each $\lambda$ in $E$,

$$
\lim _{r / 1}(1-r) h_{m}(r \lambda)=0
$$

uniformly for $m \geq 0$. Then $\sum_{n=0}^{\infty} a_{n} z^{n}=h(z)$ for each $z$ in $\Gamma \backslash E$.

Proof. By Corollary 4.4, $\left\|a_{n}\right\| \rightarrow 0$. The conclusion follows from a classical result of Fatou and Riesz [27, 7.31].

The next result appears in [19, Theorem 4], but we give a proof for completeness. A sequence $\left(b_{n}\right)_{n \geq 0}$ in $\ell^{1}\left(\mathbb{Z}_{+}\right)$is said to be of spectral synthesis with respect to a closed subset $E$ of $\Gamma$ if, for each $\varepsilon>0$, there exist a sequence $\left(c_{n}\right)_{n \in \mathbb{Z}}$ and an open subset $U$ of $\Gamma$ containing $E$, such that $\sum_{n=-\infty}^{\infty} c_{n} z^{n}=0$ for all $z$ in $U$, $\sum_{n<0}\left|c_{n}\right|<\varepsilon$ and $\sum_{n \geq 0}\left|b_{n}-c_{n}\right|<\varepsilon$.

Theorem 6.3. Let $\left(a_{n}\right)_{n \geq 0}$ be a bounded sequence in $X$, let $h(z)=\sum_{n=0}^{\infty} a_{n} z^{n}$ for $|z|<1$, and let $E$ be the singular set of $h$ in $\Gamma$. Let $\left(b_{n}\right)_{n \geq 0}$ be a sequence in $\ell^{1}\left(\mathbb{Z}_{+}\right)$which is of spectral synthesis with respect to $\bar{E}=\{\bar{\lambda}: \lambda \in E\}$. Then $\left\|\sum_{n=0}^{\infty} b_{n} a_{n+k}\right\| \rightarrow 0$ as $k \rightarrow \infty$. 
Proof. Let

$$
g(t)=b_{n} \quad(n \leq t<n+1 ; n \geq 0) .
$$

Then $g \in L^{1}\left(\mathbb{R}_{+}\right)$and $g$ is of spectral synthesis with respect to $\left\{\eta \in \mathbb{R}: e^{-i \eta} \in\right.$ $\bar{E}\}=-E^{\prime}$. By Theorem 4.7,

$$
0=\lim _{k \rightarrow \infty}\left\|\int_{0}^{\infty} g(s) f(s+k) d s\right\|=\lim _{k \rightarrow \infty}\left\|\sum_{n=0}^{\infty} b_{n} a_{n+k}\right\| .
$$

Now, let $T$ be a bounded linear operator on $X$, let $x \in X$, and suppose that $\sup _{n}\left\|T^{n} x\right\|<\infty$. In the context above, we may put $a_{n}=T^{n} x$. Then

$$
h(z)=\sum_{n=0}^{\infty} z^{n} T^{n} x=(I-z T)^{-1} x=z^{-1}\left(z^{-1} I-T\right)^{-1} x
$$

for $0<|z|<\|T\|^{-1}$.

The local unitary spectrum $\sigma_{u}(T, x)$ is the set of points $\lambda$ in $\Gamma$ such that there do not exist an open neighbourhood $U$ of $\{z \in \mathbb{C}:|z|>1\} \cup\{\lambda\}$ and a holomorphic function $g: U \rightarrow X$ with $(z I-T) g(z)=x$ for all $z \in U$. Thus the singular set of $h$ in $\Gamma$ is the conjugate of $\sigma_{u}(T, x)$. (Note that for an operator with spectral radius greater than $1, \sigma_{u}(T, x)$ may be strictly larger than the unitary part of the local spectrum in the usual sense of operator theory (see [13, XV.2.6]).) We therefore obtain the following from Theorem 6.2.

Theorem 6.4. Let $T$ be a bounded linear operator on $X, x \in X$, and suppose that $\sup _{n}\left\|T^{n} x\right\|<\infty, \sigma_{u}(T, x)$ is countable, and for each $\lambda \in \sigma_{u}(T, x)$,

$$
\lim _{r \nearrow 1}(1-r)\left\|\sum_{n=0}^{\infty} r^{n} \lambda^{-n} T^{n+m} x\right\|=0,
$$

uniformly for $m \geq 0$. Then $\left\|T^{n} x\right\| \rightarrow 0$ as $n \rightarrow \infty$.

\section{REFERENCES}

1. G.R. Allan, A.G. O'Farrell and T.J. Ransford, A Tauberian theorem arising in operator theory, Bull. London Math. Soc. 19 (1987), 537-545. MR 89c:47003

2. W. Arendt and C.J.K. Batty, Tauberian theorems and stability of one-parameter semigroups, Trans. Amer. Math. Soc. 306 (1988), 837-852. MR 89g:47053

3. W. Arendt and J. Prüss, Vector-valued Tauberian theorems and asymptotic behavior of linear Volterra equations, SIAM J. Math. Anal. 23 (1992), 412-448. MR 92m:47150

4. B. Basit, Harmonic analysis and asymptotic behavior of solutions to the abstract Cauchy problem, Semigroup Forum 54 (1997) 58-74. CMP 97:03

5. C.J.K. Batty, Tauberian theorems for the Laplace-Stieltjes transform, Trans. Amer. Math. Soc. 322 (1990), 783-804. MR 91c:44001

6. C.J.K. Batty, Some Tauberian theorems related to operator theory, Functional analysis and operator theory (J. Zemánek, ed.), vol. 30, Banach Center Publ., Warsaw, 1994, pp. 21-34. MR 95f: 44001

7. C.J.K. Batty, Asymptotic behaviour of semigroups of operators, Functional analysis and operator theory (J. Zemánek, ed.), vol. 30, Banach Center Publ., Warsaw, 1994, pp. 35-52. MR 95g: 47058

8. C.J.K. Batty, Spectral conditions for stability of one-parameter semigroups, J. Diff. Equations 127 (1996), 87-96. MR 97e:47060

9. C.J.K. Batty, J. van Neerven and F. Räbiger, Local spectra and individual stability of uniformly bounded $C_{0}$-semigroups, Trans. Amer. Math. Soc. 350 (1998), 2071-2085. 
10. C.J.K. Batty and Vũ Quôc Phóng, Stability of individual elements under one-parameter semigroups, Trans. Amer. Math. Soc. 322 (1990), 805-818. MR 91c:47072

11. R. deLaubenfels, Existence families, functional calculi and evolution equations, Lecture Notes in Math. vol. 1570, Springer, Berlin, 1994. MR 96b:47047

12. R. deLaubenfels and Vũ Quôc Phóng, Stability and almost periodicity of solutions of ill-posed abstract Cauchy problems, Proc. Amer. Math. Soc. 125 (1997), 235-241. MR 97c:34123

13. N. Dunford and J.T. Schwartz, Linear operators III, Wiley-Interscience, New York, 1971. MR 54:1009

14. J. Esterle, E. Strouse and F. Zouakia, Stabilité asymptotique de certains semigroupes d'opérateurs et idéaux primaires de $L^{1}\left(\mathbb{R}_{+}\right)$, J. Operator Theory 28 (1992), 203-227. MR 95f: 43001

15. E. Hille and R.S. Phillips, Functional analysis and semigroups, Amer. Math. Soc., Providence, 1957. MR 19:664d

16. Falun Huang, Spectral properties and stability of one-parameter semigroups, J. Diff. Equations 104 (1993), 182-195. MR 94f: 47047

17. A.E. Ingham, On Wiener's method in Tauberian theorems, Proc. London Math. Soc. 38 (1935), 458-480.

18. S. Kantorovitz, The Hille-Yosida space of an arbitrary operator, J. Math. Anal. Appl. 136 (1988), 107-111. MR 90a:47097

19. Y. Katznelson and L. Tzafriri, On power bounded operators, J. Functional Anal. 68 (1986), 313-328. MR 88e:47006

20. J. Korevaar, On Newman's quick way to the prime number theorem, Math. Intelligencer 4 (1982), 108-115. MR 84b:10063

21. S.G. Krein, G.I. Laptev and G.A. Cvetkova, On Hadamard correctness of the Cauchy problem for the equation of evolution, Soviet Math. Dokl. 11 (1970), 763-766. MR 42:637

22. U. Krengel, Ergodic theorems, De Gruyter, Berlin, 1985. MR 87i:28001

23. Yu. I. Lyubich and Vũ Quôc Phóng, Asymptotic stability of linear differential equations on Banach spaces, Studia Math. 88 (1988), 37-42. MR 89e:47062

24. H. Pollard, Harmonic analysis of bounded functions, Duke Math. J. 20 (1953), 499-512. MR 15:215f

25. W.M. Ruess and Vũ Quôc Phóng, Asymptotically almost periodic solutions of evolution equations in Banach spaces, J. Diff. Equations 122 (1995), 282-301. MR 96i:34143

26. O.J. Staffans, On asymptotically almost periodic solutions of a convolution equation, Trans. Amer. Math. Soc. 266 (1981), 603-616. MR 83b:46056

27. E.C. Titchmarsh, The theory of functions, Oxford Univ. Press, Oxford, 1932.

28. Vũ Quôc Phóng, Theorems of Katznelson-Tzafriri type for semigroups of operators, J. Functional Anal. 103 (1992), 74-84. MR 93e:47050

29. Vũ Quôc Phóng, On the spectrum, complete trajectories, and asymptotic stability of linear semi-dynamical systems, J. Diff. Equations 105 (1993), 30-45. MR 94f:47049

St. John's College, Oxford OX1 3JP, England

E-mail address: charles.batty@sjc.ox.ac.uk

Department of Mathematics, Delft Technical University, P.O. Box 356, 2600 AJ Delft, The Netherlands

E-mail address: J.vanNeerven@twi.tudelft.nl

Mathematisches Institut, Universität Tübingen, Auf Der Morgenstelle 10, D-72076 Tübingen, Germany

E-mail address: frra@michelangelo.mathematik.uni-tuebingen.de 